\title{
Photoelectron Spectra and Geometric Structures of Small Niobium Cluster Anions
}

\author{
Hardy Kietzmann, ${ }^{1}$ J. Morenzin, ${ }^{1}$ Paul S. Bechthold, ${ }^{1}$ Gerd Ganteför, ${ }^{1}$ Wolfgang Eberhardt, ${ }^{1}$ \\ Dong-Sheng Yang, ${ }^{2}$ Peter A. Hackett, ${ }^{2}$ René Fournier, ${ }^{3}$ Tao Pang, ${ }^{3}$ and Changfeng Chen ${ }^{3}$ \\ ${ }^{1}$ Institut für Festkorperforschung, Forschungszentrum Jülich, 52425 Jülich, Germany \\ ${ }^{2}$ Steacie Institute for Molecular Sciences, National Research Council of Canada, \\ 100 Sussex Drive, Ottawa, Ontario, K1A OR6, Canada \\ ${ }^{3}$ Department of Physics, University of Nevada, Las Vegas, Nevada 89154
}

\begin{abstract}
Photoelectron spectroscopy measurements and density functional theory calculations are combined to determine structures of $\mathrm{Nb}_{n}{ }^{-}(n=3-8)$ clusters. A detailed comparison between observed and calculated electronic binding energies shows that the clusters have low-symmetry compact 3D structures and the lowest possible total spin, except for the three- and five-ato clusters which are in triplet states. We fin evidence for the coexistence of two isomers of $\mathrm{Nb}_{8}{ }^{-}$under some experimental conditions. This approach shows great promise for structural characterization of small clusters.
\end{abstract}

PACS numbers: 36.40.Mr, 31.15.Ew, 33.80.Eh, 36.40.Wa

Despite widespread interest in clusters [1] and much effort, very little is known about the geometric structure of small elemental clusters in the gas phase. This is especially true for transition metal clusters. Some structures, mostly highly symmetric ones, have been proposed on the basis of ion mobilities [2] or experiments sensitive to surface sites [3]. Such studies, however, give little detail about the geometry and rest upon assumptions about the relation between geometric structure and properties (such as reactivity) that are often difficul to demonstrate. On the theoretical side, structures of many clusters have been suggested on the basis of the calculated relative energies of different isomers. There are two problems with such studies. First, except for very small clusters, there is always a danger of missing the lowest energy isomer. An extensive search with a global optimization technique can solve this problem, but only at a high computing cost which practically precludes the use of accurate electronic structure methods. Second, the energetically favored isomer is not necessarily the one formed under certain laboratory conditions: other isomers can be kinetically favored. A more direct and reliable determination of cluster structure can be made by comparing spectra, which are generally structure sensitive, to predictions of theory for different isomers [4,5]. In this paper, we present the firs systematic study that combines photoelectron spectroscopy measurements and local spin density (LSD) calculations for a transition metal cluster series, $\mathrm{Nb}_{n}{ }^{-}(n=3-8)$. The calculated relative energies for optimized geometries provide likely stable structures, and comparison of the calculated electronic binding energies (BEs) to the measured spectra, which are highly structured, allows an essential "reality check" of the theoretical prediction.

The experimental setup has been described in detail in a previous publication [6]. Niobium cluster anions are produced by laser vaporization ( $\mathrm{KrF}$ excimer laser, $5.0 \mathrm{eV}$ ) of a niobium rod in the presence of a pulse of helium gas.
The cluster anions are guided down a clustering tube and are supersonically expanded into the vacuum chamber. The cluster source is cooled with liquid nitrogen. After passing a skimmer, the anions are accelerated in a pulsed electric fiel and directed into the source region of a "magnetic bottle" time-of-fligh electron spectrometer. Depending on their velocities the anions separate into a chain of bunches of a define cluster size. A selected bunch is then decelerated in the center of the electron spectrometer and subsequently irradiated by a uv laser pulse ( $\mathrm{XeCl}$ excimer, $4.0 \mathrm{eV})$. The kinetic energy of electrons detached from the selected anions is calculated from their fligh time and calibrated using the spectrum of the $\mathrm{Cu}$ anion.

We have performed LSD calculations with the program deMon [7], using a model core potential [8] to describe the [Ar] $3 d^{10} 4 s^{2}$ inner shells and basis sets for the 11 valence electrons described and tested previously [9-11]. Many trial geometries have been optimized by minimizing the norm of the energy gradient by a standard method. The resulting geometries are characterized by calculating the force constants and vibrational frequencies. When an optimized structure is not at the energy minimum we distort it along the imaginary mode and resume geometry optimization. Our search for minima is extensive and has included structures not reported here. We will give more details, including all geometric parameters and vibrational frequencies, in a forthcoming paper [12]. The electronic BEs are calculated according to the generalized transition state (GTS) formula [13], $D_{i}^{\mathrm{GTS}}=-\left[\epsilon_{i}(1)+\right.$ $\left.3 \epsilon_{i}(1 / 3)\right] / 4$, where $\epsilon_{i}(\lambda)$ is the energy of orbital $\phi_{i}$ after a self-consistent fiel (SCF) calculation with occupation number $n_{i}$ held equal to $\lambda$. We have subtracted the ground state total energy of the neutral at the anion equilibrium geometry from that of the anion. This gives a second determination of the lowest $\mathrm{BE}$, or electron affinit (EA), $D_{a}^{\mathrm{SCF}}$. All GTS values are then shifted by $\Delta=D_{a}^{\mathrm{SCF}}-$ $D_{a}^{\mathrm{GTS}}(\Delta$ was typically about $+1.25 \mathrm{eV})$, and these shifted 
values are what we report here. As a consistency check, we also have used Slater's transition state method [14] to calculate the energy of exciting an electron from $\phi_{i}$ to $\phi_{a}$, $\Delta E_{i a}$, in the neutral cluster at the anion equilibrium geometry. We can view ionization from $\phi_{i}$ as a two-step process and set $D_{i}^{(2)}=D_{a}^{\mathrm{SCF}}+\Delta E_{i a}$. The average magnitude of the difference between $D_{i}^{(2)}$ and shifted $D_{i}^{\text {GTS }}$ is about $0.01 \mathrm{eV}$ [12].

All the calculated equilibrium structures are displayed in Fig. 1. On that basis alone, the structures of the clusters are expected to be $3 \mathrm{a}, 4 \mathrm{a}, \ldots, 8 \mathrm{a}$. As we will show below, the calculated BEs (vertical lines in Fig. 2) confir this prediction for all but the three- and five-ato clusters which we assign to structures $3 \mathrm{~b}$ and $5 \mathrm{~b}$.

Among triatomics, structure $3 \mathrm{~b}$ clearly gives the best match to experiment. The calculated EA at $1.09 \mathrm{eV}$ and BEs at 2.70 and $2.76 \mathrm{eV}$ coincide with the experimental peaks. The eight BEs in the $1.85-2.45 \mathrm{eV}$ range fall in the region where two strong and rather broad peaks are seen. Structure 3a gives a poor match: the small number (only three) of BEs in the 1.4-2.5 eV range and their position can hardly account for the two intense peaks. The EA and the $\mathrm{BE}$ nearest the $2.75 \mathrm{eV}$ peak differ from experimental data by 0.1 and $0.25 \mathrm{eV}$, respectively. Structure $3 \mathrm{c}$ is not at an energy minimum and does not match the spectrum. Our assignment ( $3 b$ ) contradicts the stability order, but the calculated relative energies of $3 a$ and $3 b$ could well be in error by more than $0.09 \mathrm{eV}$. Moreover, we cannot reject the possibility of a mixture of $3 \mathrm{~b}$ and $3 \mathrm{a}$, but $3 \mathrm{~b}$ is certainly present.

We can rule out structures $4 \mathrm{~b}$ and $4 \mathrm{c}$ considering the difference between their $D_{a}$ and the lowest energy peak $(0.45$ and $0.7 \mathrm{eV})$ and the overall distribution of BEs. The BEs of structure 4a do not match precisely the experimental data but they form a pattern consistent with the overall shape of the spectrum. In addition to structures $4 a-4 c$, we have done calculations for the square, $\mathrm{C}_{3 v}$ pyramid, and ideal tetrahedron structures. They are not minima on the potential surface. The BEs for a perfect $\left(T_{d}\right)$ tetrahedron (not shown in Fig. 2) are close to those of structure 4a but give a slightly poorer match to the experimental data. We also have obtained a partially optimized geometry of the $\mathrm{C}_{2 v}$ planar endon capped triangle. Its high energy $(4.2 \mathrm{eV})$, EA $(1.67 \mathrm{eV})$, and other BEs rule out this structure.

The best match to the $\mathrm{Nb}_{5}{ }^{-}$spectrum comes from structure $5 \mathrm{~b}, \mathrm{a} \mathrm{C}_{s} \mathrm{Jahn}-\mathrm{Teller}$ distorted trigonal bipyramid. The EA is off by $0.3 \mathrm{eV}$, but the BEs near 1.75 and $1.9 \mathrm{eV}$ can account for the large intense peak, those between 2.2 and $2.6 \mathrm{eV}$ match the second peak, and the BEs above $2.75 \mathrm{eV}$ are consistent with the onset of the continuum. Similar to $\mathrm{Nb}_{3}{ }^{-}$, the small energy difference between structures $5 \mathrm{a}$ and $5 \mathrm{~b}(0.30 \mathrm{eV})$ suggests a possible mixture of isomers. The singlet trapezoid (5c) has only one BE near $1.9 \mathrm{eV}$, and the triplet (5d) has only two, which cannot account for the broad and intense peak (suggesting many
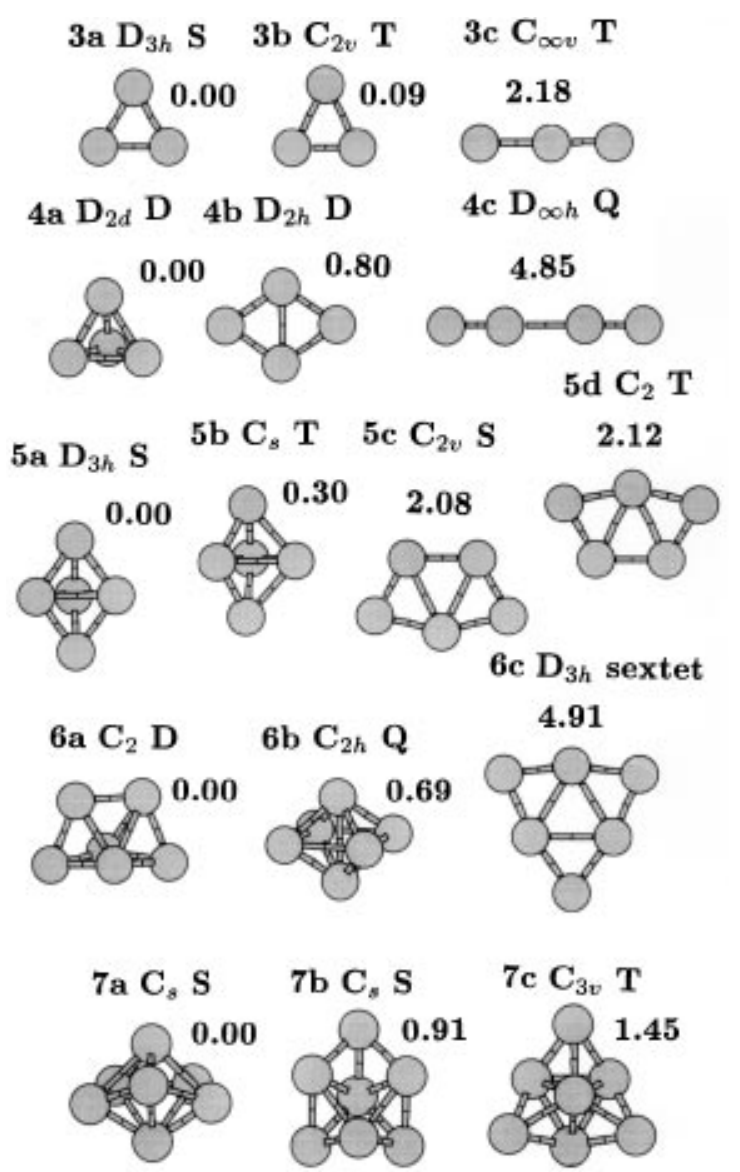

$7 \mathrm{c} \mathbf{C}_{3 v} \mathbf{T}$

$7 \mathrm{~d} \mathbf{C}_{2} \mathbf{T}$
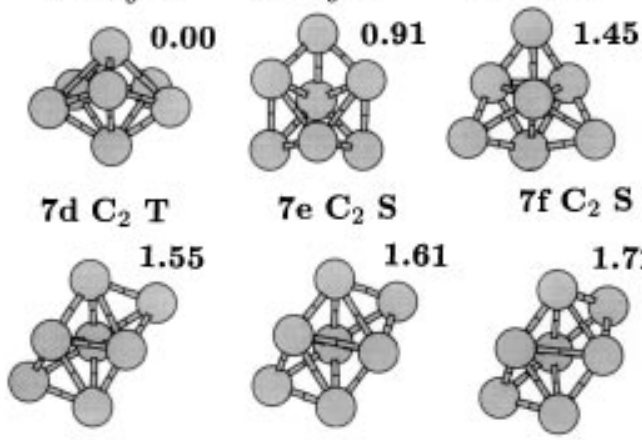

$7 \mathrm{f}_{2} \mathrm{~S}$

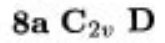

$8 \mathrm{~b} \mathrm{C} \mathrm{C}_{1} \mathbf{D}$
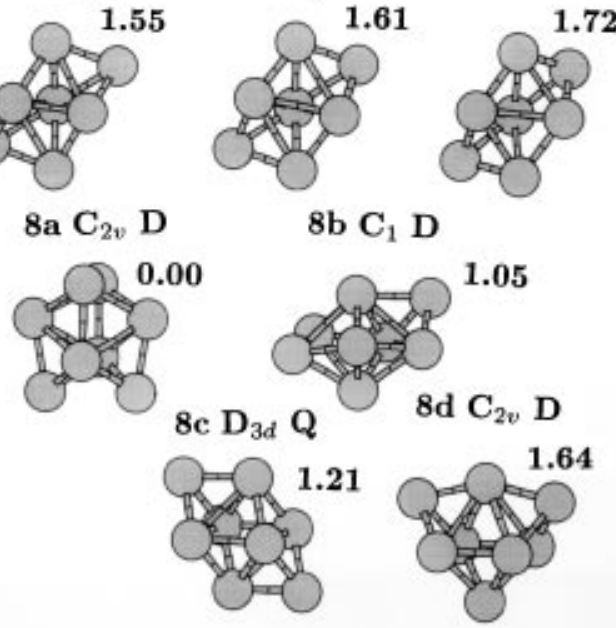

1.05

FIG. 1. The optimized $\mathrm{Nb}_{n}{ }^{-}$cluster structures, their pointgroup symmetry, spin multiplicity $(S=$ singlet, $D=$ doublet, $T=$ triplet, and $Q=$ quartet), and relative energy (in $\mathrm{eV}$ ). Bonds are drawn for atom pairs closer than $3 \AA$. All the structures shown are minima of the potential surface except $3 \mathrm{c}$ and $4 \mathrm{c}$ which are second order critical points.

transitions) observed at $1.75 \mathrm{eV}$. The square pyramid is not at an energy minimum.

The errors on $D_{a}$, relative to the experimental EA, are roughly $-0.1,-0.3$, and $+0.35 \mathrm{eV}$ for structures $6 \mathrm{a}, 6 \mathrm{~b}$, 

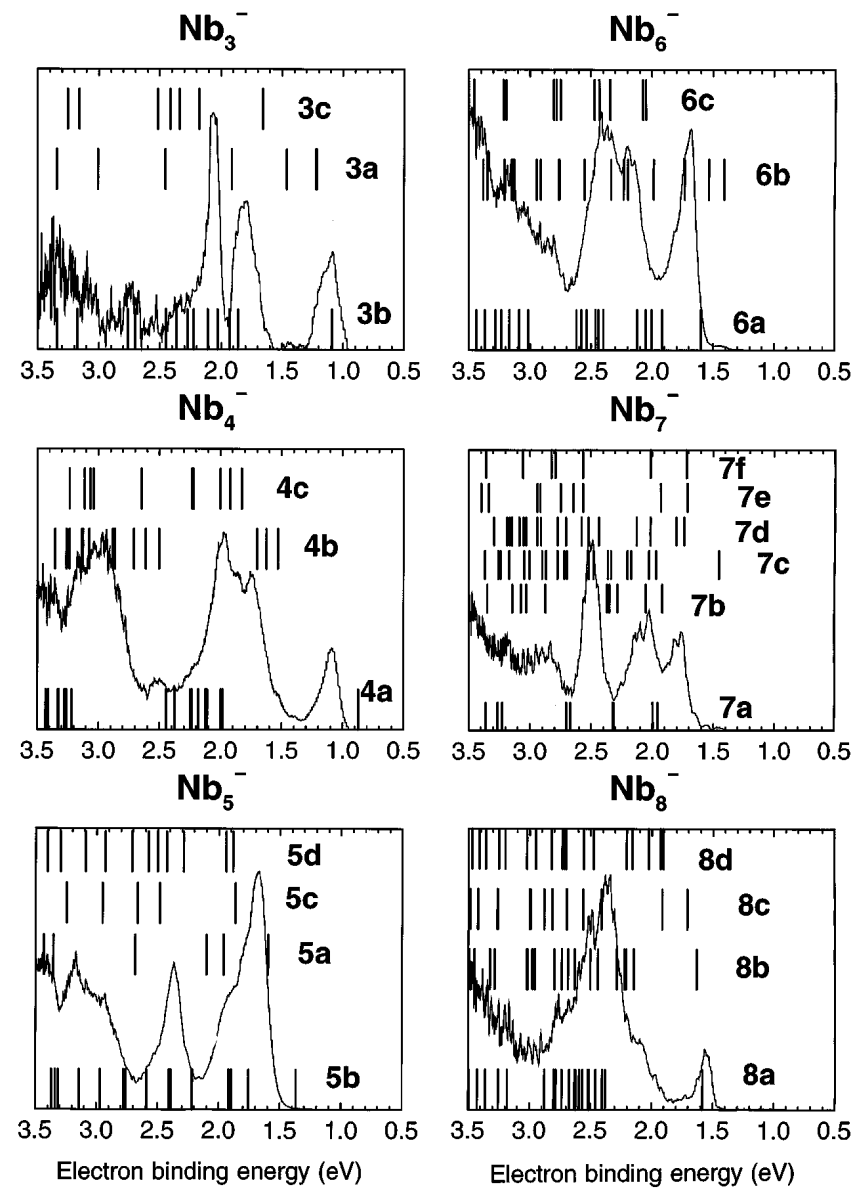

FIG. 2. Measured photoelectron spectra and calculated BEs (vertical lines) of the 3- to 8-atom niobium cluster anions. The labels 3a, 3b, ... correspond to those in Fig. 1. No peak nor calculated $\mathrm{BE}$ is found below $0.5 \mathrm{eV}$.

and $6 \mathrm{c}$, respectively. Structure $6 \mathrm{~b}$ has BEs that are never more than $0.25 \mathrm{eV}$ apart in the range from 1.4 to $3.5 \mathrm{eV}$ which implies an almost featureless spectrum. On the contrary, the BEs of $6 \mathrm{a}$ show a pattern consistent with the observed structured spectrum. There is a stationary point for the doublet state of structure $6 \mathrm{c}$, but it has two imaginary frequencies and distortion followed by further optimization makes it go back to 6 a.

The three pairs of close-lying BEs of structure $7 \mathrm{a}$, a distorted pentagonal bipyramid, match the three main peaks in the measured spectrum, although they are $0.2 \mathrm{eV}$ too high. On the contrary, the BEs of $7 \mathrm{~b}, 7 \mathrm{c}$, and $7 \mathrm{e}$ do not match the overall shape of the spectrum, even if they are shifted so as to reproduce the electron affinity The lowest BEs of $7 \mathrm{~d}$ match the spectrum nicely, but the numerous BEs between 2.7 and $3.2 \mathrm{eV}$ seem inconsistent with the low intensity in that part of the spectrum. Although $7 \mathrm{~d}$ and $7 \mathrm{f}$ have BEs that are not entirely inconsistent with the spectrum, their fairly high relative energies (1.55 and $1.72 \mathrm{eV}$ ) make them unlikely candidates for the structure of $\mathrm{Nb}_{7}$.
The $D_{a}$ of structure 8 a coincides with the lowest energy peak of $\mathrm{Nb}_{8}{ }^{-}$, and the large number of BEs in the 2.35$2.9 \mathrm{eV}$ range can account for the broad peak centered at $2.4 \mathrm{eV}$. Even the small gap in the calculated BEs (from 2.9 to $3.2 \mathrm{eV}$ ) seems to have an equivalent in the observed spectrum. The BEs of structure $8 \mathrm{~b}$ match the spectrum about as well, while the match is poor for structures $8 \mathrm{c}$ and $8 \mathrm{~d}$. We have found that the measured spectrum of $\mathrm{Nb}_{8}{ }^{-}$depends on the source condition. The spectrum of Fig. 3 was obtained by tuning the source parameters to maximize the gap between the EA peak and the main feature between 2.2 and $2.7 \mathrm{eV}$, and by using a slightly different photon energy (3.49 eV) [15]. The BEs of 8a agree almost perfectly with this spectrum while the BEs of $8 \mathrm{~b}$ do not give such a good match. Comparing the two spectra, the main peak is broader and the EA peak at $1.55 \mathrm{eV}$ is broader and asymmetrical in Fig. 2. This indicates a contribution from $8 \mathrm{~b}$ to the spectrum in Fig. 2, whereas the spectrum in Fig. 3 is almost purely due to $8 \mathrm{a}$.

The equilibrium structures of $\mathrm{Ag}_{n}{ }^{-}$clusters predicted by configuratio interaction calculations [16] differ from our proposed $\mathrm{Nb}_{n}{ }^{-}$cluster structures for $n=3$, 4, and 5 (Ref. [16] gives the $D_{\infty h}$ linear triatomic, $4 \mathrm{~b}$, and $5 \mathrm{c}$ topologies as being the most stable) but closely resemble those of $\mathrm{Nb}_{n}{ }^{-}$for $n=6,7$, and 8. Alkali metal neutral clusters adopt planar structures [17] while $\mathrm{Na}_{3}{ }^{-}, \mathrm{Na}_{4}{ }^{-}$, $\mathrm{K}_{4}{ }^{-}$, and also possibly $\mathrm{Na}_{5}{ }^{-}$, are predicted to be linear [18]. On the contrary, the neutral $\mathrm{Nb}_{n}$ clusters are predicted to be compact [9], and the anions structures we have found here are just as compact. This is due to the open $d$ shells of $\mathrm{Nb}$ atoms which lead to strong multiple bonds (the $\mathrm{Nb}_{n} \rightarrow \mathrm{Nb}_{n-1}+\mathrm{Nb}$ dissociation energies are in the 5-7 eV range [19]) and favor high coordination. In Fig. 1, bonds are drawn for atom pairs with distance

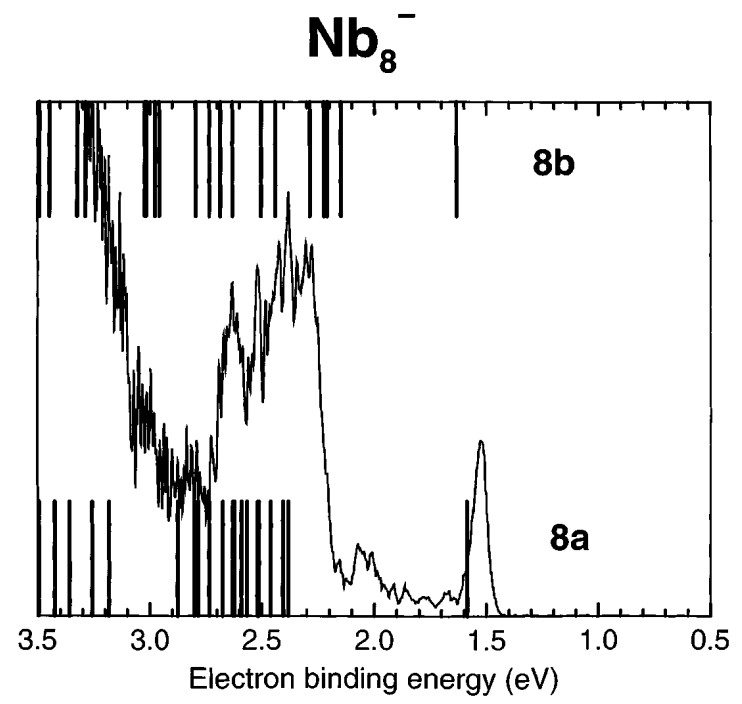

FIG. 3. $\mathrm{Nb}_{8}{ }^{-}$spectrum recorded under conditions different (see text) from the one in Fig. 2. The BEs of $8 \mathrm{a}$ and $8 \mathrm{~b}$ are shown as vertical lines. 
smaller than $3 \AA$. If we defin the mean coordination $\bar{n}$ as twice the number of bonds divided by cluster nuclearity, we see that the equilibrium structures tend to maximize $\bar{n}$. However, this rule is not strict: structures $6 \mathrm{a}$ and $8 \mathrm{a}$ have the next highest $\bar{n}$ within their size category, and fiv 7 atom clusters have the same $\bar{n}$ despite energy differences up to $1.7 \mathrm{eV}$.

The calculated equilibrium structures of niobium cluster anions are related to those of the neutrals [9], but with symmetry lowering distortions in two cases: from $T_{d}$ to $D_{2 d}$ in $\mathrm{Nb}_{4}$, due to Jahn-Teller effect, and from $\mathrm{C}_{2 v}$ to $\mathrm{C}_{2}$ in $\mathrm{Nb}_{6}$. None of the structures we have found is highly symmetric. This contrasts with the predictions from calculations using classical potentials or effective medium theory, which are often the highest possible symmetry structures. However, the spectra of $\mathrm{Nb}_{4}{ }^{-}$and $\mathrm{Nb}_{7}{ }^{-}$ are about equally well explained by the $T_{d}$ and $D_{5 h}$ structures [12]. In general, establishing the symmetry of the equilibrium structure of clusters is difficul because of contributions from nontotally symmetric vibrational modes to the thermal broadening.

We conclude that the $\mathrm{Nb}_{n}{ }^{-}$clusters we observed have structures $3 \mathrm{~b}, 4 \mathrm{a}, 5 \mathrm{~b}, 6 \mathrm{a}, 7 \mathrm{a}$, and $8 \mathrm{a}$, with possible admixtures of $3 a, 5 a$, and $8 b$ (see Fig. 1). We have found evidence that, depending on cluster source conditions, $\mathrm{Nb}_{8}{ }^{-}$ is either purely isomer $8 \mathrm{a}$ or a mixture of $8 \mathrm{a}$ and $8 \mathrm{~b}$. To our knowledge, this makes $\mathrm{Nb}_{n}{ }^{-}(n=3-8)$ the firs transition metal cluster series for which there is such detailed and reliable structural information. We want to emphasize that cluster anion photodetachment experiments and density functional theory calculations of BEs presented here are not limited to a few specifi cases; they can be readily applied to a wide range of clusters of size up to roughly ten atoms $[5,18,20]$. Therefore, besides our finding for $\mathrm{Nb}_{n}{ }^{-}$, this work shows that photoelectron spectroscopy combined with theory is a very promising approach for structural characterization of small clusters.

Work at University of Nevada, Las Vegas, was supported in part by the NSF Grant No. OSR-9353227, the DOE EPSCoR program, and the W. M. Keck Foundation.

[1] See, for example, articles on clusters in 16 February 1996 issue of Science.
[2] M. F. Jarrold, J. Phys. Chem. 99, 11 (1995), and references therein.

[3] W. F. Hoffman III, E. K. Parks, and S. J. Riley, J. Chem. Phys. 90, 1526 (1989); E. K. Parks and S. J. Riley, J. Chem. Phys. 99, 5898 (1993); E. K. Parks, L. Zhu, and S. J. Riley, J. Chem. Phys. 102, 7377 (1995); E. K. Parks and S. J. Riley, Z. Phys. D 33, 59 (1995); E. K. Parks, L. Zhu, and J. Ho, J. Chem. Phys. 100, 7206 (1994); J. Ho, E. K. Parks, and S. J. Riley, Chem. Phys. 201, 245 (1995).

[4] J. Blanc, V. Bonačić-Koutecký, M. Broyer, J. Chevaleyre, P. Dugourd, J. Koutecký, C. Scheuch, J.P. Wolf, and L. Wöste, J. Chem. Phys. 96, 1793 (1992).

[5] R. O. Jones, G. Ganteför, S. Hunsicker, and P. Pieperhoff, J. Chem. Phys. 103, 9549 (1995).

[6] H. Handschuh, G. Ganteför, and W. Eberhardt, Rev. Sci. Instrum. 66, 3838 (1995).

[7] A. St-Amant and D. R. Salahub, Chem. Phys. Lett. 169, 387 (1990); A. St-Amant, Thèse de doctorat, Université de Montréal, 1992.

[8] J. Andzelm, E. Radzio, and D. R. Salahub, J. Chem. Phys. 83, 4573 (1985).

[9] L. Goodwin and D. R. Salahub, Phys. Rev. A 47, R774 (1993).

[10] A. M. James, P. Kowalczyk, R. Fournier, and B. Simard, J. Chem. Phys. 99, 8504 (1993).

[11] D.-S. Yang, M.Z. Zgierski, D. M. Rayner, P. A. Hackett, A. Martinez, D. R. Salahub, P.-N. Roy, and T. Carrington, J. Chem. Phys. 103, 5335 (1995).

[12] R. Fournier, T. Pang, and C. Chen (to be published).

[13] A. R. Williams, R.A. deGroot, and C.B. Sommers, J. Chem. Phys. 63, 628 (1975).

[14] J. C. Slater, Phys. Rev. B 81, 385 (1951); Adv. Quantum Chem. 6, 1 (1972).

[15] At standard source conditions the spectrum recorded at $h \nu=3.49 \mathrm{eV}$ is almost identical to the one recorded at $h \nu=4.0 \mathrm{eV}$. A higher laser intensity is available at $3.49 \mathrm{eV}$ photon energy.

[16] V. Bonačić-Koutecký, L. Češpiva, P. Fantucci, J. Pittner, and J. Koutecký, J. Chem. Phys. 100, 490 (1994).

[17] J. Koutecký and P. Fantucci, Chem. Rev. 86, 539 (1986).

[18] V. Bonačić-Koutecký, P. Fantucci, and J. Koutecký, J. Chem. Phys. 93, 3802 (1990).

[19] D. A. Hales, L. Lian, and P. B. Armentrout, Int. J. Mass Spectrom. Ion Process. 102, 269 (1990).

[20] C. Massobrio, A. Pasquarello, and R. Car, Phys. Rev. Lett. 75, 2104 (1995); B. K. Rao, P. Jena, and A. K. Ray, Phys. Rev. Lett. 76, 2878 (1996). 